\title{
Trabalho em equipe na atenção primária: a experiência de Portugal
}

\author{
Marize Barros de Souza, ${ }^{1}$ Paulo de Medeiros Rocha, ${ }^{2}$ \\ Armando Brito de Sá3 e Severina Alice da Costa Uchoa²
}

Como citar Souza MB, Rocha PM, Sá AB, Uchoa SAC. Trabalho em equipe na atenção primária: a experiência de Portugal. Rev Panam Salud Publica. 2013;33(3):190-5.

RESUMO Objetivo. Analisar o trabalho em equipe na atenção primária em saúde em Portugal.

Métodos. Foi realizada uma pesquisa avaliativa de abordagem qualitativa, com desenho de estudo de caso. Os dados foram obtidos por entrevista semiestruturada, observação direta e análise documental. Foram entrevistados gestores, profissionais e usuários de 11 unidades de saúde familiar (USF) portuguesas, totalizando 71 participantes. Utilizou-se a técnica de análise de conteúdo na interpretação das entrevistas.

Resultados. Cada equipe era constituída por médico, enfermeiro e funcionário administrativo, atendendo entre 1250 e 2060 usuários. Uma característica marcante da experiência portuguesa foi a formação das equipes nas USF de forma voluntária, por meio de afinidades pessoais, com autonomia de composição. Observou-se nessas USF o desenvolvimento de "carteira básica de serviços" juntamente com intervenções de vigilância, promoção da saúde e prevenção de doença, cuidados em situação de doença aguda, acompanhamento clínico de doença crônica e de patologia múltipla, cuidados domiciliares, interligação e colaboração em rede com outros serviços (cuidados hospitalares). Foram relatadas dificuldades no atendimento domiciliar. A informatização era ampla nas USF. De acordo com os entrevistados, as mudanças advindas da implementação das USF foram maior acessibilidade dos usuários aos serviços, maior qualidade do cuidado prestado e trabalho em equipe com objetivos e metas, além da existência de um plano de ação.

Conclusões. Mesmo não tendo assumido um papel de coordenação da rede de cuidados, a atenção primária em saúde organizada a partir de equipes foi avaliada positivamente em Portugal, como promotora de maior acesso, continuidade e humanização dos serviços.

Palavras-chave Atenção primária à saúde; relações interprofissionais; Brasil; Portugal.

A atenção primária em saúde tem sido definida como um conjunto de valores e princípios que servem para orientar o desenvolvimento dos sistemas de saúde. Na Europa, o estabelecimento desse con-

\footnotetext{
1 Universidade Federal do Rio Grande do Norte (UFRN), Escola de Enfermagem, Natal (RN), Brasil. Correspondência: marizebs@gmail.com

2 UFRN, Departamento de Saúde Coletiva, Natal (RN), Brasil.

3 Universidade de Lisboa, Faculdade de Medicina, Instituto de Medicina Preventiva, Lisboa, Portugal.
}

junto de valores e princípios é dificultado pela diversidade dos sistemas de saúde existentes $(1,2)$. Uma questão central para os países europeus na atualidade é a melhora da eficiência do setor e das respostas aos constrangimentos, sobretudo econômicos (1-4).

A visão da atenção primária em saúde como coordenadora de redes de atenção na Europa $(2,4,5)$ mereceu destaque em documento da Organização Mundial da Saúde (OMS) (6). Nessa análise, a situação de Portugal se destaca pela alta cobertura vacinal e pela crescente queda nos índices de mortalidade infantil. Nesse sentido, os cuidados de saúde primários (CSP) em Portugal passaram, desde 2006, por uma extensa reforma organizacional, com a criação das Unidades de Saúde Familiar (USF). As USF passaram a ser a base de desenvolvimento da prestação de cuidados de saúde a uma determinada população, com autonomia de gestão técnico- 
assistencial e funcional, trabalhando em rede e próximas dos cidadãos (7).

As equipes portuguesas são compostas por três a 10 médicos de família, tendo igual número de enfermeiros de família e de profissionais administrativos, com cobertura populacional variando entre 4000 e 17000 pessoas. Além disso, as equipes recebem incentivos financeiros e profissionais que recompensam o mérito e são sensíveis a produtividade, acessibilidade e qualidade da atenção (7).

No Brasil, por sua vez, a experiência da Estratégia Saúde da Família (ESF) teve início com o Programa Saúde da Família (PSF) e foi orientada pela tentativa de mudança do modelo assistencial a partir da atenção primária em saúde. Diversos estudos sobre estruturas, processos e resultados da atenção primária em saúde têm recebido destaque no cenário nacional nos últimos anos, notadamente a partir de 2003, com o Projeto de Expansão do Programa Saúde da Família (PROESF) $(8,9)$. Tais análises foram realizadas a partir de distintas dimensões, com estudos de caso em várias regiões do país e em grandes centros urbanos (10-16).

Portugal e Brasil são países com fortes laços históricos, políticos e culturais. $\mathrm{Na}$ área da atenção primária em saúde, trocas de experiência e intercâmbios científicos têm sido desenvolvidos. Portugal é um dos países europeus que mais desenvolveu instrumentos de avaliação para utilização em CSP junto aos centros de saúde. O Brasil faz esse reconhecimento ao utilizar a ferramenta portuguesa de Monitorização da Qualidade Organizacional dos Centros de Saúde, MoniQuOr (17).

$\mathrm{Na}$ atualidade brasileira, o Ministério da Saúde (18) considera o trabalho em equipe como elemento-chave na área da atenção primária em saúde. Como consequência, houve crescimento e ampliação das equipes pela criação dos Núcleos de Apoio à Saúde da Família (NASF), a partir de 2008 (19). Dados recentes mostram uma forte expansão da atenção primária por meio da ESF, com cerca de 30000 equipes e 236000 agentes comunitários de saúde em atuação, com atendimento a 98 milhões de brasileiros, alcançando 85\% (4 737) dos municípios existentes (20). Estudos de caso sobre a ESF destacam os elementos diferenciais do trabalho em equipe na atenção primária em saúde no Brasil $(21,22)$.

Em 2008, o Conselho Nacional de Desenvolvimento Científico e Tecnológico
(CNPq) brasileiro apoiou um estudo para avaliar a implantação das USF no processo de reforma dos CSP em Portugal (23), com coordenação conjunta de pesquisadores brasileiros e portugueses. Para isso, foram priorizados os aspectos processuais de análise, com utilização dos instrumentos experimentados em investigações brasileiras $(8,12)$. O objetivo do presente artigo é apresentar os resultados obtidos por essa pesquisa quanto ao trabalho em equipe nos CSP em Portugal. Para isso, foram selecionados, como elementos centrais de análise e discussão, quatro aspectos do trabalho em equipe: perfil dos profissionais, processo de trabalho da equipe, ações desenvolvidas e mudanças nas práticas de cuidado.

\section{MATERIAIS E MÉTODOS}

O presente artigo descreve os resultados de uma pesquisa avaliativa $(24,25)$, de natureza qualitativa (26), desenhada como caso descritivo (27). Foram utilizadas as seguintes dimensões de análise: político-institucional, organização da atenção e organização do cuidado integral. Cada dimensão agrupou diversas subdimensões (categorias), dentro de um marco lógico-teórico já utilizado em outras pesquisas no Brasil $(8,12,15$, $16,23,28)$.

Para o presente artigo, o "trabalho em equipe" foi considerado uma subdimensão do cuidado. Essa subdimensão foi avaliada pela composição multiprofissional, definição de responsabilidades e mecanismos de integração (cooperação, reuniões de equipe, plano de trabalho, sobrecargas e resistências ao trabalho em equipe) $(23,28)$.

Foram realizados ajustes na matriz dimensional para inclusão de instrumentos validados anteriormente em pesquisa brasileira $(8,10)$ e para a realização de estudo-piloto, em abril de 2008, em Lisboa, no intuito de testar os instrumentos a serem utilizados. Em seguida, realizou-se o trabalho de campo, entre maio e outubro de 2008, em 11 USF distribuídas nas cinco administrações regionais de saúde existentes no país.

Em relação às técnicas, foram realizadas entrevistas semiestruturadas com gestores dos conselhos diretivos das administrações regionais de saúde, coordenadores das USF e profissionais e usuários das equipes de saúde familiar. Além disso, realizaram-se observação direta nas USF, com aplicação de roteiro de coleta de informações, e análise documental. Foi utilizada ainda a estratégia de triangulação $(26,29)$, ou seja, uma combinação de técnicas e procedimentos que integra as diferentes formas de avaliação.

Quanto à seleção dos participantes, deu-se de forma intencional, com prioridade à inclusão de todos os cinco gestores das administrações regionais de saúde e dos 11 coordenadores das USF selecionadas, num total de 16 entrevistas. Os critérios para a escolha das USF foram o tempo de implantação das equipes (priorizaram-se as mais antigas, constituídas entre 2006 e 2007) e a localização geográfica (litoral ou interior) dessas unidades (23).

Para a seleção dos profissionais, foi utilizado o critério geral da proporcionalidade na composição das equipes (um médico, um enfermeiro, um funcionário administrativo). Assim, houve um total de 33 entrevistas nas 11 USF selecionadas. Com relação aos usuários, foram selecionados dois usuários por USF, em virtude das limitações operacionais da investigação (tempo e custos), o que totalizou 22 entrevistas. Foram critérios de inclusão dos usuários o registro como usuário na USF e a participação nas ações programáticas mais destacadas (saúde infantil, controle de hipertensão e diabetes, planejamento familiar) (23).

Na interpretação dos dados, utilizouse a técnica de análise de conteúdo (30). O estudo foi aprovado pela Comissão de Ética da Faculdade de Medicina da Universidade de Lisboa. Os participantes assinaram um termo de consentimento informado.

\section{RESULTADOS}

\section{Perfil das equipes}

As equipes das USF eram constituídas por médico, enfermeiro e administrativo. Havia entre seis e oito equipes em cada unidade. Observou-se uma cobertura assistencial entre 7500 a 16500 usuários por USF, ou seja, entre 1250 e 2060 usuários por equipe de trabalho. No perfil dos profissionais, houve um predomínio, principalmente entre os médicos, de idades mais avançadas (entre 41 e 60 anos).

Em relação ao tempo de atuação nos CSP, observamos um predomínio de profissionais com entre 1 a 11 anos de 
experiência, embora com presença significativa daqueles com mais de 12 anos de experiência. Outra constatação é que a maioria desses profissionais $(71,4 \%)$ residia na área de abrangência das USF. Quanto ao tempo de formação, predominaram os profissionais com 20 a 36 anos de formação acadêmica.

Sobre as capacitações, observou-se que a maioria possuía cursos (especializações, residências) na área de saúde familiar, ressaltando-se que há obrigatoriedade de residência em medicina familiar/enfermagem de comunidade para os profissionais. Em relação a cursos de atualização mais específicos dos CSP, mais de $90 \%$ tinham formação em planejamento familiar, diabetes e hipertensão; $81 \%$ realizaram capacitação em áreas específicas dos ciclos de vida (saúde materna e infantil).

Uma característica com forte destaque observada na experiência portuguesa foi a formação das equipes de trabalho nas USF de forma voluntária, por meio de afinidades pessoais e com autonomia de composição, com uma formação do tipo bottom-up (23) (caráter participativo, ascendente, a partir dos profissionais no nível local das unidades de saúde).

\section{Processo de trabalho da equipe}

A avaliação identificou uma percepção favorável por parte dos entrevistados, que ressaltou a existência de integração e cooperação entre os membros da equipe, com maior socialização das informações, plano de trabalho comum, existência de reuniões para decisões coletivas e planejamento participativo, destacando-se ainda o respeito pelas diferenças. Essa avaliação favorável também foi constatada na observação direta das USF. Exemplos concretos dessa verificação in loco são as ótimas condições de trabalho encontradas nessas unidades de saúde, com infraestrutura apropriada ao pleno desenvolvimento das equipes e de suas ações (instalações físicas de boa qualidade arquitetônica, com espaços bem definidos; equipamentos básicos e materiais de consumo em situação adequada, com manutenção e abastecimento regulares). Também foi constatada a disponibilidade de medicamentos básicos, de uso regular na unidade.

Com relação à sobrecarga de trabalho, constatou-se a existência dessa situação entre os enfermeiros. Isso ocorreu devido à necessidade de demissão de alguns profissionais que não se adequavam às exigências do trabalho com $\mathrm{O}$ novo modelo nos CSP, embora a resistência ao trabalho em equipe não tenha sido significativa entre os profissionais.

Outro aspecto ressaltado pelos profissionais foi a necessidade de melhorar as reuniões com estratégias voltadas para a interação da equipe. Ao responderem sobre as razões da adesão dos profissionais e sobre os aspectos positivos da reforma dos CSP, o trabalho em equipe apareceu como destaque do processo. Nas reuniões de equipe, ainda é incipiente a participação de usuários ou de representantes das comunidades.

Percebeu-se que o trabalho em equipe impulsionava fortemente outros aspectos das atividades nas USF. Nesse sentido, surgiram como elementos destacados pelos profissionais, gestores e usuários, respectivamente, a motivação no desenvolvimento das ações, a maior responsabilidade nos cuidados e a melhoria na satisfação da atenção recebida.

Além disso, a equipe tinha autonomia nas questões técnico-operacionais. Esse foi um elemento de destaque nas respostas dos gestores e profissionais, demonstrando melhorias na capacidade de resolução das equipes nos trabalhos dos CSP.

\section{Ações desenvolvidas}

As USF desenvolviam ações específicas, uma "carteira básica de serviços" (31) que se constitui no seu compromisso assistencial nuclear. Verificou-se ainda que as equipes realizavam inúmeras atividades e intervenções nas grandes áreas de serviços clínicos: vigilância, promoção da saúde e prevenção de doença nas diversas fases da vida, cuidados em situação de doença aguda, acompanhamento clínico de doença crônica e de patologia múltipla, cuidados nos domicílios (visitas), interligação e colaboração em rede com outros serviços (cuidados hospitalares).

Quanto às ações educativas em saúde, observou-se que eram direcionadas prioritariamente para grupos de hipertensão e diabetes, gestantes e planejamento familiar, pessoas com obesidade, idosos, prevenção dos cânceres do colo uterino e de mama e saúde infantil. Com menor frequência, observaram-se trabalhos educativos com adolescentes e tabagismo, prevenção de doenças sexualmente transmissíveis e HIV, alcoo- lismo e tuberculose. Ações relacionadas à saúde escolar, oral e ocupacional, e outros cuidados continuados integrados ficavam sob a responsabilidade de outras unidades funcionais de saúde, como as Unidades de Cuidados na Comunidade.

Todos os profissionais relataram dificuldades para desenvolver visitas domiciliares. Quando ocorriam, essas visitas tinham um enfoque clínico-curativo, com atendimento de usuários impossibilitados de locomoção. A grande maioria dos usuários informou não receber visita dos profissionais de saúde.

No que diz respeito à informatização no trabalho, as equipes, regularmente, utilizavam dados dos sistemas de informação em saúde no planejamento de suas ações, na referência de pacientes, no registro de atividades, como também no seu monitoramento e avaliação. A informatização ampla nas USF foi uma realidade destacada na experiência portuguesa, com inovação tecnológica e criação recente de sistema informático próprio (23). Da mesma forma, verificouse a utilização regular de protocolos de atenção (clínico-terapêuticos) em áreas básicas de atuação das equipes (vacinação, saúde infantil, planejamento familiar e saúde materna, diabetes mellitus).

\section{Mudanças nas práticas de cuidado}

Os profissionais destacaram mudanças nas práticas do cuidado, sendo as mais referidas: maior acessibilidade dos usuários aos serviços, qualidade do cuidado prestado e trabalho em equipe com objetivos e metas, além da existência de um plano de ação. Em destaque, a liberdade de escolher uma equipe para participação surgiu como fator de motivação para o novo trabalho, com maior garantia de continuidade e humanização nas práticas de cuidado.

Quanto às dificuldades, foram destacadas questões relacionadas ao processo de trabalho - "vícios e hábitos profissionais arraigados" (descumprimento de horários, longo tempo de espera e falta de entendimento dos usuários sobre a nova organização do trabalho). Além disso, foram encontradas dificuldades relativas às questões técnico-administrativas, a saber: falhas no sistema de informação, que dificultam o seu melhor aproveitamento; deficiências na articulação com os hospitais; falta de autonomia administrativa para compras; incertezas e indefinições político-institucionais sobre 
a reforma dos CSP; descumprimento de incentivos profissionais.

Entre os gestores, foram apontadas como facilidades dos CSP: maior acessibilidade; trabalho em equipe (a partir de sua formação voluntária) e qualidade da atenção. Para os coordenadores de USF, a consulta de enfermagem surgiu como elemento inovador nessas práticas. Sobre as dificuldades, foram destacadas questões relativas aos sistemas de informação, falta de formação específica sobre gestão de saúde e resistências dentro da administração pública.

Entre os usuários, predominaram comentários relacionados à satisfação com $\mathrm{o}$ atendimento recebido, destacando a figura do profissional médico, a equipe de trabalho (enfermagem e administrativos) e a acessibilidade aos serviços (alargamento de horários). Importante registrar as insatisfações verificadas no que diz respeito às dificuldades de agendamento para os médicos de família (referiram haver um número alto de pessoas a serem atendidas por esse profissional), bem como o tempo de espera para a marcação de consultas.

\section{DISCUSSÃO}

Na experiência portuguesa, as USF, de acordo com o presente estudo, funcionam com uma equipe mínima (três integrantes) e com padrões de cobertura assistencial que guardam coerência com as linhas prioritárias de ação preconizadas (31). No Brasil, ao contrário, predominam equipes com número maior de integrantes (entre 10 a 12): médicos, enfermeiros, técnicos de enfermagem, agentes comunitários de saúde, dentistas e técnicos em saúde bucal.

Sobre as capacitações dos profissionais, observaram-se diferenças também significativas: em Portugal, há exigência de formação prévia em atenção primária em saúde; no Brasil, ao contrário, não há obrigatoriedade dessa formação (residências, especializações) na área de saúde familiar. Entretanto, percebe-se a existência de variados mecanismos compensatórios a essas insuficiências na experiência brasileira, com uma diversificada oferta de cursos: especialização em saúde da família, curso introdutório à ESF (obrigatório), capacitações nos ciclos de vida, vacinação, doenças crônicas. A partir de 2005, foi definido no Brasil um Programa Nacional de Reorientação da Formação Profissional em Saúde (PRÓ-
SAÚDE), visando à transformação do processo de formação, com ênfase na atenção básica em saúde. Em 2009, essa perspectiva foi ampliada com a criação do Programa de Educação pelo Trabalho para a Saúde (PET-SAÚDE), que objetiva uma maior integração dos cursos de graduação em saúde às necessidades da atenção básica.

No Brasil, há dificuldade de fixar médicos na atenção primária em saúde. A realidade em algumas regiões do país é a do médico recém-formado, sem residência ou especialização, que pretende permanecer apenas temporariamente na atenção primária em saúde. Não reside na localidade e, por isso, não está presente diariamente na USF, o que compromete $\mathrm{o}$ vínculo com a equipe e a população. Assim, outra iniciativa governamental diz respeito ao Programa de Valorização da Atenção Básica (PROVAB), cujo objetivo é levar médicos, dentistas e enfermeiros para municípios que têm dificuldade de contratar esses profissionais, particularmente pequenos municípios das regiões Norte e Nordeste.

Diferentemente de Portugal, as equipes da ESF no Brasil são constituídas de forma involuntária, a partir da ordem de classificação dos seus integrantes no processo seletivo. Isso tem ocasionado problemas de integração, o que traz dificuldades para o processo de trabalho na atenção primária em saúde brasileira.

Quanto ao processo de trabalho das equipes, aspectos como plano de trabalho unificador de ações, sobrecargas e resistências, reuniões coletivas (tomadas de decisão) e respeito pelas diferenças podem ser considerados semelhantes em ambos os países. Também é semelhante a avaliação positiva na percepção dos entrevistados $(9,13,20,21)$. Em ambos os casos, o trabalho em equipe contribuiu, de forma decisiva, para uma maior humanização dos serviços nas USF, com melhoria do acesso aos usuários (23), que se traduz no avanço da qualidade dos cuidados prestados.

Com relação à sobrecarga de trabalho, existem diferenças entre as experiências. Enquanto em Portugal concentra-se na enfermagem, no Brasil existe uma sobrecarga generalizada para a equipe, em virtude da demanda excessiva que frequentemente ultrapassa o padrão preconizado pelo Ministério da Saúde (1 000 famílias por equipe).

Um aspecto decisivo para a avaliação do trabalho em equipe encontra-se na verificação das condições de trabalho nas USF. Em Portugal, as melhores condições de infraestrutura contribuem, inegavelmente, para um bom desempenho das equipes e sua autonomia técnicogerencial. No Brasil, existem sérias dificuldades nessas condições de infraestrutura da ESF: espaços físicos inadequados, déficit de material, equipes incompletas. Tais fatores têm comprometido o acesso e a qualidade da atenção prestada, o que levou o Ministério da Saúde a criar o Programa de Melhoria do Acesso e Qualidade da Atenção Básica (PMAQ-AB) (32).

Pode-se afirmar que as experiências portuguesa e brasileira na atenção primária em saúde guardam identidade nas ações desenvolvidas pelas equipes de saúde. Em Portugal, existem outros serviços no próprio âmbito dos cuidados primários em saúde (serviços responsáveis pelas atividades educativas), restringindo assim as ações das USF à parte assistencial. No Brasil, ao contrário, as equipes da ESF são responsáveis pela totalidade das ações assistenciais e preventivo-promocionais. Isso tem ocasionado dificuldades, levando o Ministério da Saúde a rever as composições dessas equipes no sentido de ampliá-las (33).

Já em relação às ações de visita domiciliar, constata-se fragilidade na experiência portuguesa, com dificuldades dos profissionais para exercerem essa atividade. No Brasil, observam-se dificuldades quanto ao deslocamento das equipes e à sobrecarga de atendimento, principalmente do médico. Ressalta-se, no Brasil, a existência do Agente Comunitário de Saúde (ACS), que atua como elo entre a comunidade e a equipe. Esse profissional é responsável pela seleção das pessoas que necessitam da visita domiciliar. Em ambos os países, predomina o enfoque clínico-curativo, com atendimento a usuários impossibilitados de locomoção.

Sobre a utilização dos sistemas de informação nas ações das equipes, observa-se o uso regular para o planejamento e o registro de atividades nas experiências estudadas. Em Portugal, verifica-se uma informatização maior desse trabalho (atividades clínicas e administrativas), enquanto que, no Brasil, apesar da disseminação do Sistema de Informação Básica (SIAB), ainda persistem dificuldades na informatização (acesso e regularidade dos instrumentos). Da mesma forma, existem semelhanças na utilização regular de protocolos de atenção (clínico-terapêuticos). 
No Brasil, pesquisas atuais evidenciam queda na taxa de mortalidade infantil neonatal, associada à redução das mortes por doenças diarreicas e por infecções respiratórias (20, 34-37). Ademais, a melhoria na satisfação dos usuários também está indicada nessas pesquisas (14). Em Portugal e no Brasil, as dificuldades de consolidação da atenção primária em saúde como coordenadora da atenção traduzem um modelo em transição ou um processo em construção.

Apesar da existência de limitações metodológicas (contextos distintos, períodos de investigação, padronização de instrumentos etc.) que tornam as discussões e suas conclusões passíveis de questionamento, foi possível dialogar com as experiências vivenciadas pelos dois países (com trabalho conjunto de pesquisadores brasileiros e portugueses), o que possibilitou o alcance dos objetivos pretendidos. Recomenda-se a realização de outras investigações de natureza comparativa, com os necessários ajustes metodológicos.

\section{Conclusões}

A implantação das equipes de saúde primária em Portugal foi bem-sucedida, pelo menos até o momento da pesquisa, apesar de limitações como a insuficiência da atenção primária em saúde como coordenadora da rede de cuidados. Tanto em Portugal como no Brasil, merecem destaque os avanços obtidos: a avaliação positiva quanto às práticas de atenção primária em saúde, com evidências de maior acesso aos serviços, continuidade e humanização, salientando-se o trabalho em equipe como elemento central nessas mudanças.

Conflito de interesses. Nada declarado pelos autores.

\section{REFERÊNCIAS}

1. Mannion R. General practitioner commissioning in the English National Health Service: continuity, change, and future challenges. Int J Health Serv. 2008;38(4):717-30.

2. Mladovsky $P$, Allin $S$, Masseria $C$, HernándezQuevedo C, McDaid D, Mossialos E. Health in the European Union: trends and analysis. Copenhague: World Health Organization on behalf of the European Observatory on Health Systems and Policies; 2009. [Observatory studies series no. 19].

3. Schlette S, Lisac M, Blum K. Integrated primary care in Germany: the road ahead. Int J Integr Care. 2009;9:e14.

4. Magnussen J, Vrangbaek K, Saltman R. Nordic Health Care Systems: recent reforms and current policy challenges. Maidenhead: Open Univerity Press; 2009. [Nordic Health Care Systems: recent reforms and current policy challenges]. Disponível em: http://www. euro.who.int/document/e93429.pdf Acessado em março de 2013.

5. Saltman R, Rico A, Boerma W. Atenção Primária conduzindo as Redes de Atenção à Saúde: reforma organizacional na atenção primária européia. Maidenhead: Open University Press; 2006. Disponível em: http:// www.google.com.br/url?sa=t\&rct=j\&q=\&esr $\mathrm{c}=\mathrm{s} \&$ source $=$ web\&cd $=1 \&$ ved $=0$ CC 4 QFjAA\& url=http $\% 3 \mathrm{~A} \% 2 \mathrm{~F} \% 2 \mathrm{Fportal}$ saude.gov.br $\% 2$ Fportal\%2Farquivos\%2Fpdf\%2Fatenc_prim cond_redes_aten_a_saude_2010.pdf\&ei $=\mathrm{k} \bar{C}$ dGUȲDjB7S60AHBOYGADw\&usg=AFQjC NEiHqbEgcTzj75WoKyK8JCENod9eg\&bvm =bv.43828540,d.eWU\&cad=rja Acessado em março de 2013.

6. Organização Mundial da Saúde (OMS). Relatório Mundial de Saúde 2008: cuidados de saúde primários - agora mais que nunca. Lisboa: Organização Mundial da Saúde e Alto Comissariado para a Saúde; 2008. Disponível em: http://www.who.int/whr/2008/ whr08_pr.pdf Acessado em março de 2013

7. Portugal. Resolução do Conselho de Ministros 157/2005. Vol. 157: Diário da República, 2005. Disponível em: http://www.mcsp. min-saude.pt/Imgs/content/page_106/ Resolucao_Conselho_Ministros_157_2005.pdf Acessado em março de 2013.
8. Rocha PM, Uchoa AC, Souza EC, Rocha ML, Escoda SQ, Rocha NS. Projeto de Expansão e Consolidação Saúde da Família (PROESF) Estudo de linha de base (pesquisa avaliativa): Lote 1 - Nordeste (21 municípios da Bahia, Ceará e Sergipe). Natal: EDUFRN; 2008.

9. de Sousa MF, Hamann EM. Programa Saúde da Família no Brasil: uma agenda incompleta? Cien Saude Colet. 2009;14 Suppl 1:1325-35.

10. Escorel SC, Giovanella L, Mendonça MH, Magalhães R, Senna MC. Saúde da Família: avaliação da implementação em dez grandes centros urbanos - síntese dos principais resultados. $2^{\mathrm{a}}$ ed. Brasília: Editora do Ministério da Saúde; 2005.

11. Viana ALA, Novaes HMD, Silva JA, Rocha JSY, Ibañez N, Elias PEM, organizadores. Caracterização dos municípios paulistas com população superior a 100 mil habitantes: avaliação do Programa de Expansão e Consolidação da Saúde da Família. São Paulo: CEDEC, Consórcio Medicina USP; 2006.

12. Rocha Pde M, Uchoa Ada C, Rocha Nde S, Souza EC, Rocha Mde L, Pinheiro TX. Uma avaliação do Programa de Saúde da Família em municípios do Nordeste do Brasil: velhos e novos desafios. Cad Saude Publica. 2008;24 Suppl 1:S69-78.

13. Conill EM. Um modelo histórico e conceitual sobre a Atenção Primária à Saúde: desafios para a organização da atenção básica e da Estratégia Saúde da Família em grandes cidades brasileiras. Cad Saude Publica. 2008;24 Suppl 1:S7-16; discussion S17-27.

14. Giovanella L, de Mendonca MH, de Almeida $\mathrm{PF}$, Escorel S, Senna Mde C, Fausto MC et al. Saúde da família: limites e possibilidades para uma abordagem integral de atenção primária de cuidados de saúde no Brasil. Cien Saude Colet. 2009;14(3):783-94.

15. Medina MG, Hartz ZM. The role of the Family Health Program in the organization of primary care in municipal health systems. Cad Saude Publica. 2009;25(5):1153-67.

16. Hartz ZM, Felisberto E, Silva LM. Metaavaliação da atenção básica à saúde. Teoria e prática. Rio de Janeiro: FIOCRUZ; 2008.

17. Brasil, Ministério da Saúde. Avaliação para melhoria da qualidade da estratégia saúde da família: documento técnico. Brasília: Ministério da Saúde; 2005. (Série B - Textos básicos de saúde).

18. Brasil, Ministério da Saúde. Atenção básica e à saude da família (equipes de saúde). Brasília: Editora DAB; 2008.

19. Brasil. Portaria 154 de 24 de janeiro de 2008. Cria os Núcleos de Apoio à Saúde da Família - NASF. Disponível em: http://bvsms. saude.gov.br/bvs/saudelegis/gm/2008/ prt0154_24_01_2008.html Acessado em março de 2013.

20. Paim J, Travassos C, Almeida C, Bahia L, Macinko J. O sistema de saúde brasileiro: história, avanços e desafios. Lancet. 2011; 377(9779):1778-97.

21. Araujo MB, Rocha Pde M. Trabalho em equipe: um desafio para a família Consolidação da Estratégia Saúde da Família. Cien Saude Colet. 2007;12(2):455-64.

22. Araujo MB, Rocha Pde M. Saúde da família: mudando práticas? Um estudo de caso na cidade de Natal (RN), Brasil. Cien Saude Colet. 2009;14 Suppl 1:1439-1452.

23. Rocha PM, Sá AB. Reforma da Saúde Familiar em Portugal: avaliação da implantação. Cien Saude Colet. 2011;16:2853-2863.

24. Contandriopoulos A, Champagne F, Denis JL, Pineault R. A avaliação na área da saúde: conceitos e métodos. Em: Hartz ZM, org. Avaliação em saúde dos modelos conceituais à prática na análise da implantação de programas. Rio de Janeiro: FIOCRUZ; 1997. Pp. 29-48.

25. Vieira-da-Silva LM. Conceitos, abordagens e estratégias para a avaliação em saúde. Em: Hartz ZM, Vieira-da-Silva LM, eds. Avaliação e saúde: dos modelos teóricos à prática na avaliação de programas e sistemas de saúde. Rio de Janeiro/Salvador: FIOCRUZ/EDUFBA, 2008. Pp. 15-40.

26. Denzin NK, YS L. Introdução: a disciplina e a prática da pesquisa qualitativa. Em: Denzin NK, Lincoln YS, eds. O planejamento da pesquisa qualitativa: teorias e abordagens. Porto Alegre: Artmed/Bookman; 2006. Pp. 15-41.

27. Yin RK. Estudo de caso: planejamento e métodos. 3a ed. Porto Alegre: Bookman; 2005.

28. Pontes G, Figueró AC. Linha de base matricial da implementação do Proesf. Em: Hartz ZM, 
Felisberto E, Silva LM, eds. Meta-avaliação da atenção básica à saúde. Teoria e prática. Rio de Janeiro: FIOCRUZ, 2008. Pp. 71-100.

29. Minayo MC, Assis SG, Souza EC. Avaliação por triangulação: abordagens de programas sociais. Rio de Janeiro: FIOCRUZ, 2005.

30. Bardin L. Análise de conteúdo. $4^{\mathrm{a}}$. ed. Lisboa, 2008.

31. Portugal. Ministério da Saúde. missão para os cuidados de saúde primários. Cuidados continuados integrados, carteira de serviços, Lisboa, 2007. Disponínvel em: http://www. mcsp.min-saude.pt/Imgs/content/page 46/ Cuidados\%20Continuados\%20nos\%20CSP. pdf Acessado em 17 de março de 2013.

32. Brasil. Portaria GM 1654 de 19 de julho de 2011. Institui o Programa Nacional de Melhoria do Acesso e da Qualidade da Atenção Básica (PMAQ-AB) e o Incentivo Financeiro do PMAQ-AB. Disponível em: http://www. brasilsus.com.br/legislacoes/gm/1088141654.html Acessado em março de 2013.
33. Brasil, Ministério da Saúde. Diretrizes do NASF (Núcleo de Apoio à Saúde da Família). Brasília: Secretaria de Atenção à Saude; 2009. [Cadernos de Atenção Básica, no. 27]. Disponível em: http://www.google.com.br/url ? sa $=\mathrm{t} \& \mathrm{rct}=\mathrm{j} \& \mathrm{q}=\&$ esrc $=\mathrm{s} \&$ source $=$ web $\& \mathrm{~cd}=1$ $\&$ cad=rja\&ved=0CC4QFjAA\&url=http $\% 3 \mathrm{~A} \%$ 2F\%2Fbvsms.saude.gov.br\%2Fbvs\%2Fpublic acoes $\% 2$ Fcaderno atencao basica diretrizes nasf.pdf\&ei=aTJGUcOnPOPX0QHL54HoDw \&usg=AFQjCNFZ IPvcNBAVEEn8I4pxITz8 2cXVg\&bvm=bv.43828540,d.dmQ Acessado em março de 2013.

34. Almeida PF, Giovanella L, Mendonça MH, Escorel S. Desafios à coordenação dos cuidados em saúde: estratégias de integração entre níveis assistenciais em grandes centros urbanos. Cad Saude Publica. 2010;26:286-98.

35. Macinko J, Marinho de Souza Mde F, Guanais FC, da Silva Simoes CC. Going to scale with community-based primary care: an analysis of the family health program and infant mortality in Brazil, 1999-2004. Soc Sci Med. 2007:65(10):2070-80.

36. Aquino R, de Oliveira NF, Barreto ML. Impact of the family health program on infant mortality in Brazilian municipalities. Am J Public Health. 2009;99(1):87-93.

37. Rasella D, Aquino R, Barreto ML. Impact of the Family Health Program on the quality of vital information and reduction of child unattended deaths in Brazil: an ecological longitudinal study. BMC Public Health. 2010;10:380.

Manuscrito recebido em 1 de fevereiro de 2012. Aceito em versão revisada em 5 de outubro de 2012.

ABSTRACT Objective. To analyze the work of health care teams delivering primary care in Portugal.

Methods. We performed an evaluative research project, with a qualitative case

Teamwork in primary care: the experience of Portugal tion, and analysis of documents. We interviewed managers, workers, and users in 11 family health units (USF) in Portugal, for a total of 71 participants. Content analysis was used to assess the interviews.

Results. Teams included a physician, a nurse, and an administrative employee. Each team was in charge of 1250 to 2060 users. A striking characteristic of the Portuguese experience was the voluntary and autonomous setup of teams at the USF, based on personal affinity. The services provided at the USF included a basic "service package" as well as activities of surveillance, health promotion, disease prevention, care of acute disease, clinical follow-up of patients with chronic or multiple diseases, home care, and networking with other services (hospital care). Difficulties in providing home care were reported. Electronic systems were widely available. According to interviewees, the changes resulting from the implementation of USF included improved user access to services, higher quality of care, and goal-oriented teamwork, guided by an action plan.

Conclusions. Even without a coordinating role in the health care network, the delivery of primary health care through teams was positively evaluated in Portugal as promoting increased access, continuity, and humanization of health services.

Key words Primary health care; interprofessional relations; Brazil; Portugal. 